\title{
DOS PROCESSOS AOS PRODUTOS DE INVESTIGAÇÃO: TRÊS \\ PROPOSTAS DE AVALIAÇÃO DE ESTUDOS CIENTÍFICOS NÃO \\ POSITIVISTAS
}

\section{FROM PROCESSES TO RESEARCH PRODUCTS: THREE PROPOSALS OF EVALUATION OF NON-POSITIVISTIC SCIENTIFIC STUDIES}

\begin{abstract}
Sónia Mairos Ferreira ${ }^{1}$
Resumo: O presente artigo constitui uma reflexão crítica acerca dos desafios e possibilidades que se colocam, na contemporaneidade, à avaliação da qualidade de investigação científica, quando esta se desenvolve segundo orientações de matriz não positivista. No âmbito da prossecução deste propósito a primeira rubrica contém uma breve síntese das (in)suficiências, reportadas na literatura, no que respeita ao positivismo e, também, às propostas alternativas defendidas pelos/as seus/suas críticos/as. A rubrica seguinte sintetiza três tendências de apreciação da qualidade de estudos científicos não positivistas, apontando as particularidades que assumem, assim como as divergências que possuem, em relação ao positivismo e, igualmente, entre si.
\end{abstract}

Palavras-chave: Conhecimento científico; Investigação (não) positivista; Avaliação.

\begin{abstract}
This article is a critical reflection on the challenges and opportunities that arise, in contemporaneity, to the evaluation of the quality of scientific research, when it develops accordingly to non-positivist guidelines. In pursuing this purpose the first rubric contains a brief overview of the (in)sufficiencies, reported in the literature, regarding positivism and, also, to alternative proposals advocated by its critics. The following rubric synthesizes three trends of assessing the quality of nonpositivist scientific studies, pointing out the particularities they assume, as well as the major differences from positivism and from each other.
\end{abstract}

Keywords: Scientific knowledge; (not) Positivist research; Evaluation.

\section{Introdução}

Another of the qualities of science is that it teaches the value of rational thought as well as the importance of freedom of thought; the positive results that come from doubting that the lessons are all true. You must here distinguish especially in teaching - the science from the forms or procedures that are sometimes used in developing science. It is easy to say, "We write, experiment, and observe, and do this or that." You can copy that form exactly. But great religions are dissipated by following form without remembering the direct content of the teaching of the great leaders. In the same way, it is possible to follow form and call it science, but that is pseudo-science. In this way, we all suffer from the kind of tyranny we have today in the many institutions that have come under the influence of pseudoscientific advisers. (...) Science alone of all the subjects contains within itself the lesson of the danger of belief in the infallibility of the greatest teachers in the preceding generation (FEYNMAN, 1969, s.p.).

\footnotetext{
1 Doutora em Ciências da Educação pela Faculdade de Psicologia e de Ciências da Educação da Universidade de Coimbra (FPCEUC), Portugal. Coordenadora da Divisão M da Associação Portuguesa Conversas de Psicologia (APCdP), Coimbra, Portugal. Email: smairosferreira@gmail.com
} 
Emergiu, no seio do profuso debate acerca do conhecimento científico de matriz não positivista, em Ciências Sociais e Humanas, questionamento especificamente devotado à avaliação da qualidade dos estudos desenvolvidos (DENZIN; LINCOLN, 2000; HESSE-BIBER; LEAVY, 2004). Neste âmbito, embora seja consensual a consideração de que qualquer iniciativa que se constitui como um contributo para o conhecimento científico está sujeita à apreciação crítica, os/as investigadores/as têm manifestado maior dificuldade em selecionar as dimensões e/ou os critérios considerados mais ajustados para efetivar essa intenção (WAGNER; LUKASSEN; MAHLENDORF, 2010). Pese o investimento consagrado à análise dos pressupostos conceptuais e metodológicos, à sistematização de procedimentos, bem como à elencagem de referentes para avaliação, as propostas desenvolvidas à data ainda não obtiveram, por parte da(s) comunidade(s) científica(s), o reconhecimento que os seus proponentes decerto aspirariam (AMADO, 2013; FERREIRA, 2015).

Note-se, contudo, que não resolução desta dificuldade não decorre, em exclusivo, de limitações intrínsecas às propostas efetuadas. As possibilidades avançadas assomaram num período de desenvolvimento do conhecimento científico em que permanece sem solução uma indagação mais complexa e profunda. Trata-se do estatuto atribuído ao conhecimento científico obtido a partir de tradições não positivistas, o qual ainda não atingiu, em muitas esferas de produção e uso de ciência, o patamar de conhecimento científico legítimo. Questionado na sua validade, adequação, sustentabilidade e, até, na sua relevância o conhecimento que não cumpre as premissas do positivismo lógico (frequentemente apontado na literatura como conhecimento resultante de "investigação quantitativa"), é tido como inferior, i.e. como uma possibilidade que deve ser remetida para pesquisas de cariz exploratório, de aproximação a um domínio, que se caraterizam pela superficialidade, maior incerteza e menor rigor.

Este artigo pretende constituir um contributo para o aprofundamento da reflexão crítica sobre o conhecimento científico reconhecendo, para o efeito, a multiplicidade de possibilidades que ciência encerra. Reconhece-se, portanto, que diversas orientações ontológicas, epistemológicas e, também, metodológicas podem ser mobilizadas, em detrimento de uma posição universalista. Consubstanciado nesta premissa, o presente trabalho de análise abraça o repto de expor alguns desafios e possibilidades que se colocam à avaliação da qualidade da investigação científica de matriz não positivista. A primeira rubrica é dedicada à perscrutação de potencialidades e insuficiências presentes no paradigma positivista de ciência. Afloram-se, em complemento, potenciais 
alternativas, salientando as suas divergências mais salientes em relação a este paradigma. Apontam-se, no ponto subsequente, três propostas diferentes de sistematização de critérios de avaliação da qualidade da investigação científica, sempre que esta se sustenta em abordagens de matriz não positivista. Trata-se, em suma, de um estudo de revisão da literatura, de fontes primárias e secundárias sobre esta temática.

\section{Conhecimento científico: (In)suficiências (im)previstas das abordagens (não) positivistas}

Vários autores alertam para o domínio que o positivismo exerce no pensamento científico, na contemporaneidade (GLAZIER; POWELL, 1992; FEYERABEND, 1993; LAW, 2006; AMADO, 2013; FERREIRA, 2015). Bogdan e Biklen (2013) assinalam, num comentário específico a esta questão, que a investigação, tal como é (re)conhecida publicamente, é sinónimo de investigação quantitativa, i.e., investigação desenvolvida segundo a orientação do positivismo. Mantêm-se, assim, preponderantes os procedimentos nele defendidos, nomeadamente no que concerne ao privilégio de abordagens dedutivas, e à centralidade da quantificação, requisitos essenciais à obtenção de conhecimento objetivo, i.e., conhecimento que possibilita o teste de generalizações hipotéticas (FEYERABEND, 1993; MERTENS, 1998; DENZIN; LINCOLN, 2000; HESSE-BIBER; LEAVY, 2004; AMADO, 2013). Toma-se como não discutível a existência de uma realidade externa, passível de ser objetivada através do recurso a instrumentos estandardizados, seja esta respeitante ao mundo físico, social ou humano.

Este paradigma privilegia o exame de relações causais entre variáveis, na tentativa de identificar um conjunto de causas que, de forma direta ou através de efeitos indiretos (e.g., de mediação), provocam consequências passíveis de ser identificadas e mensuradas. O/a investigador/à deve delimitar o fenómeno que pretende estudar, sistematizando-o segundo um conjunto finito de elementos categorizados de forma quantitativa, sendo estes utilizados como traduções objetivas e numéricas de realidades tão díspares como grupos, pessoas, situações, objetos ou, mesmo, órgãos do corpo humano. Perante a impossibilidade de estudar o universo que seleciona, pede-se ao/à cientista que dele recolha uma amostra, preferivelmente a partir de procedimentos aleatórios (WINTER, 2000; PATTON, 2001).

Neste paradigma o investigador/a é, em simultâneo, humano e não-humano, dado que é capaz de se despir das suas crenças, convicções, dúvidas e inquietações, dos seus 
sentimentos, ..., ou seja dos atributos que o constituem enquanto ser único (neste âmbito tidos como imperfeições), transmutando-se num ser vivo, mas racional, neutro e objetivo, que investiga sem (se) influenciar, objetos de estudo que podem ser escrutinados a partir das partes que os constituem, segundo perguntas antecipadamente formuladas, e de acordo com as regras de tradução (da realidade externa estudada para o documento escrito a ser produzido) que o conhecimento científico consente. Qualquer referência que possa indiciar não neutralidade por parte do/a investigador/a constitui uma séria limitação ao estudo realizado.

A ciência possui, portanto, um vocabulário próprio, no qual os conceitos problema, hipóteses, amostra, representatividade, aleatorização, testes, medidas, controlo, experimental, protocolo, estandardização assumem claro protagonismo. Vemolos repetidos, com regularidade, em trabalhos científicos, grande parte dos quais sem qualquer esforço no sentido da sua análise crítica ou, mesmo, sem qualquer proposta de clarificação do seu significado (FEYERABEND, 1993; RAGIN, 2008). Como referem Bogdan e Biklen (2013), a sua utilização é de tal forma comum que já não é questionada a sua significação, relevância e/ou adequação, ainda que nem sempre saibamos o que estes termos significam, num determinado estudo. Em contraste, a adjetivação deve ser abolida, assim como o uso de termos coloquiais, de alegorias, metáforas ou outras figuras de estilo. As emoções desaparecem, excepto se estas constituírem o objeto de estudo, ou seja, se integrarem parte ou o próprio universo em análise. O/a investigador/a positivista não teme, não entristece, não regozija, não se irrita nem se perturba enquanto realiza investigação. É perpetuamente neutro/a, sob pena de enviesar os resultados e a sua interpretação.

Em oposição aos pressupostos referidos emergiram, no seio das Ciências Sociais e Humanas, propostas alternativas de investigação, as quais comungam entre si o propósito de combater a hegemonia do pensamento positivista (DENZIN; LINCOLN, 2000; HESSE-BIBER; LEAVY, 2004; RAGIN, 2008; AMADO, 2013). Reunidas sob a designação comum de "investigação qualitativa", tendo em consideração que uma das suas maiores comunalidades reside no privilégio conferido a metodologias de tipo nãoquantitativo (GOLAFSHANI, 2003, ROLFE, 2006), estas abordagens não-positivistas não são, contudo, facilmente unificáveis se considerarmos as diferenças epistemológicas, ontológicas ou até metodológicas, que possuem entre si. Em resultado desta diversidade, a investigação qualitativa diz respeito, salvo exceções documentadas de forma explícita, a estudos que não foram efetuados a partir dos pressupostos disseminados pelo paradigma 
positivista de ciência.

Em estreita interdependência com o exposto, investigação de tipo qualitativo tem vindo a ser caracterizada não com base nos elementos que lhe conferem especificidade, mas sim a partir daquilo que ela não é (GLAZIER; POWELL, 1992). Esta preterição dos fundamentos ontológicos e epistemológicos dos trabalhos não positivistas provocou um efeito de simplificação das opções assumidas pelos/as cientistas sociais, que passam a ser sustentadas pela assunção da premissa da não-aceitação do positivismo (PATTON, 2001; SILVERMAN, 2013). Assim, ainda que sejam recorrentes as alusões às divergências epistemológicas, ontológicas e metodológicas em relação ao positivismo, estas não são explanadas numa elevada percentagem dos trabalhos científicos produzidos. Simplificase, portanto, um debate complexo, que passa a circunscrever-se às opções metodológicas assumidas pelos/as investigadores/as. Como refere Partington (2000, p. 92), “questões ontológicas (o que é considerado realidade) e epistemológicas (como pode ser estabelecido o conhecimento da realidade) são frequentemente evitados de forma ardilosa, assumidos como algo garantido ou ignorados".

Veja-se, ainda em registo ilustrativo do que temos vindo a referir, o elevado número de estudos em que surge referência explícita à não manipulação de variáveis, complementado com alusão ao propósito de compreensão dos fenómenos em contexto, em detrimento da explicação e da intenção de generalizar os resultados, argumentos utilizados para defender o recurso à não quantificação dos dados (AMADO, 2013; FERREIRA, 2015). Neste sentido situa-se, precisamente, a afirmação avançada por Strauss e Corbin (1998, p. 17), que definem investigação qualitativa como todo o tipo de investigações em que os resultados não são obtidos através de procedimentos estatísticos ou qualquer estratégia de quantificação de dados.

O método de recolha e análise dos dados passa, então, a constituir-se como elemento distintivo da tradição não positivista que o/a autor/a defende. Abraçam esta posição autores como Glazier e Powell (1992), Sandelowski e Barroso (2002) ou Rolfe (2006) que chamam a atenção para as ambiguidades existentes em diversos trabalhos publicados, assinalando a imprescindibilidade de clarificação das premissas epistemológicas, ontológicas e, até, metodológicas que suportam o estudo.

As críticas às abordagens não positivistas não se circunscrevem, contudo, a esta dimensão. Designados como "soft scientists", os/as investigadores/as que perfilham abordagens de matriz não positivista são considerados/as, por muitos dos seus pares, como produtores de trabalhos pseudo-científicos, de cariz exploratório, com influência 
de fatores pessoais e com enviesamentos que minam a sua credibilidade (AMADO, 2013). Fragilidades que têm vindo a ser apontadas como evidência de que a qualidade das investigações qualitativas é menor (WAGNER; LUKASSEN; MAHLENDORF, 2010; SILVERMAN, 2013).

\section{A apreciação da qualidade de estudos científicos não positivistas}

Assumindo que o conhecimento científico se abre à análise crítica, mesmo quando este é realizado a partir de abordagens com especificidades muito diferentes entre si, é imprescindível que se descrevam, de forma clara e transparente, os procedimentos de avaliação que orientam a tomada de decisão sobre a qualidade dos estudos (STENBACK, 2001; DAVIS; DODD, 2002; FERREIRA, 2015). Contudo, e pese o incremento do investimento no âmbito da clarificação dos elementos que sustentam estarmos perante uma investigação que se pode incluir no conjunto de trabalhos que compõe "ciência válida", à data ainda não existe um conjunto consensualizado de indicadores, passível de uso no âmbito da avaliação da qualidade de um estudo não positivista. A afirmação de Sandelowski e Barroso (2002) permanece, pois, muito atual, na medida em que, de facto, não estamos sequer próximos do nível de consenso necessário para que se possa considerar que temos um conjunto sistematizado de critérios de utilização em estudos de matriz qualitativa.

Cientistas que perfilham o positivismo, no âmbito das Ciências Sociais e Humanas, defendem o recurso aos critérios utilizados de forma generalizada pelas ciências exatas (ROLFE, 2006). Para esta linha de investigadores/as o recurso a referenciais comuns, tidos em conta de forma independente dos pressupostos (epistemológicos, ontológicos e metodológicos) do estudo tornaria viável a comparação entre projetos e produções científicas muito heterogéneos. Propõe-se, portanto, universalidade em relação aos procedimentos, critérios e indicadores de apreciação da qualidade dos trabalhos científicos produzidos, sendo esta universalidade assumida a partir dos parâmetros defendidos pelo positivismo (AMADO, 2013; FERREIRA, 2015).

Em clara discordância com a proposta anterior, autores que perfilham abordagens não positivistas tendem a não adoptar os critérios defendidos pelo paradigma dominante. Esta não aceitação constitui um ato de resistência, uma vez que se pretende assinalar as diferenças entre tradições de investigação, reforçando a necessidade de que diversas abordagens sejam reconhecidas na sua singularidade (FERREIRA, 2015). As propostas 
alternativas abraçam a convicção de que as Ciências Sociais e Humanas devem libertarse da ortodoxia metodológica de que ainda são reféns (LINCOLN; GUBA, 1985; CORBIN; STRAUSS, 1990; DENZIN; LINCOLN, 2000; STENBACKA, 2001). Propõem, de entre várias possibilidades, que o processo de apreciação da qualidade da investigação realizada se situe, não no/a investigador/a - responsável pelo acesso e recolha de informação/dados relevante(s), mas sim nos/as participantes e leitores/as do estudo - a quem cabe a apreciação da confiança do estudo (ROLFE, 2006).

O percurso e os processos de investigação devem estar explícitos, assegurandose a clareza e a simplicidade necessárias para que estes/as últimos/as possam posicionarse em relação à qualidade do estudo desenvolvido. Para os/as defensores/as desta proposta a qualidade dos estudos deriva da confiabilidade (trustworthiness), em substituição do escrutínio da (não) aproximação dos resultados à verdade. Não se aspira uma possível generalização dos resultados, nem o assegurar da validade e fidelidade dos resultados, tal como defendido pelo positivismo. Em contraste atende-se à natureza contextual e heurística das investigações, assumindo como exequível, e defensável, o recurso a possibilidades diversas de avaliação da qualidade das investigações efetuadas (AMADO, 2013).

Sem intenções de exaustividade trazemos alguns exemplos desta segunda linha de orientação, apontando as suas caraterísticas e principais virtualidades e fragilidades. Glaser e Strauss $(1965,1967)$ apontam, na sua reflexão, como critérios comuns, a densidade, a integração, a clareza, a abrangência, a parcimónia, a consistência e, por fim, a lógica. Para Lincoln e Guba (1985) a avaliação da qualidade da investigação assenta na apreciação da confiabilidade (trustworthiness). Para a sua concretização propõem quatro critérios, que foram sugeridos com o intuito de constituírem alternativas viáveis aos critérios tradicionalmente mobilizados no seio do paradigma positivista, a saber: (i) credibilidade (credibility), (ii) confirmação (confirmability); (iii) dependência (dependability), e, por último, (iv) transferência (transferability).

O primeiro destes critérios, credibilidade, é obtido quando se verifica que os resultados apresentados são concetualizados como plausíveis por parte daqueles/as que colaboraram no estudo. Este critério corresponde, em investigação positivista, ao critério de validade interna (LINCOLN; GUBA, 1985; GUBA; LINCOLN, 1998). Por sua vez, a confirmação possui paralelo com o critério de objetividade. Este critério respeita ao grau de possível confirmação dos resultados e das leituras interpretativas efetuadas pelo/a cientista, por parte de outros indivíduos. Tendo em consideração que a neutralidade do/a 
investigador/a é uma impossibilidade, pois estamos perante pessoas não privadas de subjetividades, este referente remete para a apreciação que outros/as fazem acerca da sua perspetiva/leitura dos dados recolhidos. O terceiro critério, dependência, diz respeito à replicação da investigação, mais específico à comparação dos resultados obtidos noutros estudos, quando se mobilizam procedimentos idênticos. Por último, a transferabilidade prende-se com a generalização dos resultados a uma população maior, possuindo evidente relação com o critério positivista de validade externa. Ao grupo referido acresce, ainda e segundo Wagner, Lukassen e Mahlendorf (2010), o elemento aplicabilidade. Este considera a apreciação do ajuste entre (i) questão de partida e objetivos e (ii) o recurso a método(s) específico(s).

Embora seja uma referência muito comum em fontes primárias e secundárias sobre metodologia de investigação, a proposta de Lincoln e Guba (1985) não se encontra, no entanto, isenta de críticas. Sandelowski (1993) salienta que o recurso a um critério que se aproxima à fidelidade (na matriz positivista) é desajustado no âmbito da investigação não-positivista. Segundo esta cientista, em estudos que não perfilham a consideração de que existe uma realidade exterior ao/à pesquisador/a, não é ajustado assumir que, perante a replicação do método, se obtenham resultados idênticos. Defende, em complemento, que também não é imprescindível que os/as participantes cheguem a conclusões análogas à do/a investigador/a. Para Sandelowski (1993), o recurso a estes critérios pode constituir uma ameaça, pois na procura de amplificá-los o/a cientista pode impor consensos artificiais e paralelismos nos dados e na sua análise, minando a validade do estudo. Defende, em complemento, que é necessário não restringir a avaliação da qualidade dos estudos aos procedimentos técnicos efetuados, incluindo elementos respeitantes às opções teóricas. Na sua perspetiva a avaliação inclui sempre uma dimensão de julgamento, que não deve ser invisibilizada, mas sim assumida e clarificada.

A última tendência assume uma proposta revolucionária sugerindo que se abandonem todas as lógicas positivistas, incluindo a de que existe universalidade no que respeita aos procedimentos de análise e avaliação da qualidade da investigação científica (FEYERABEND, 1993; LAW, 2006). A indicação é a de que se rejeite a determinação apriorística de critérios (HOPE; WATERMAN, 2003), cabendo ao/à investigador/a a decisão acerca dos procedimentos e dos indicadores que mobilizará no estudo que efetua. A pluralidade é, neste âmbito, não apenas assumida como também defendida como requisito básico da implementação de abordagens que pretendem um conhecimento científico de matriz não universalista e não determinista (FEYERABEND, 1993; LAW, 
2006; RAGIN, 2008).

\section{Considerações finais}

Apesar da diversidade de argumentos que atestam as limitações inerentes ao paradigma positivista de ciência, particularmente no que respeita ao seu contributo para as ciências sociais e humanas, este permanece como uma referência incontornável no debate sobre ciência e, em específico, sobre a produção de conhecimento científico. Seja devido à anuência por parte de um conjunto ainda muito elevado de cientistas ou, resultante, da sua não aceitação, este constitui como marco incontornável, pelo qual se avalia a qualidade dos estudos desenvolvidos em diversos quadrantes. Neste âmbito, embora se reconheça uma crescente diversidade de pressupostos epistemológicos, ontológicos e metodológicos, os estudos não desenvolvidos de acordo com o paradigma positivista continuam a ser alvo de reservas e suspeita por parte da comunidade científica.

Contudo, as insuficiências deste paradigma têm vindo a ser notabilizadas, sendo hoje evidente que este não permite dar conta da complexidade que o conhecimento científico pretende abraçar. Emergem e fortalecem-se, assim, visões alternativas, que se sedimentam em concepções epistemológicas, ontológicas e metodológicas heterogéneas. A riqueza de perspetivas é, na contemporaneidade, um elemento central nos debates sobre ciência e conhecimento científico, sendo profusos os contributos de investigadores/as que combatem a dominação do pensamento positivista, em defesa de uma ciência que se constrói a partir de multíplices contributos, que aceita visões complementares, analogias e, também, antagónicas, e que se revela capaz de refletir criticamente sobre si própria e sobre os seus pressupostos, propósitos, discursos e métodos.

$\mathrm{Na}$ primeira componente deste artigo afloramos alguns dos elementos que caraterizam as abordagens positivistas, contrastando-os com propostas alternativas de conceção e concretização de investigação científica. Apontamos, ainda, as fragilidades e insuficiências, não apenas do paradigma dominante (positivista), mas também das vulnerabilidades evidenciadas pelos/as seus/suas críticos/as. Conforme referimos, a diversidade de propostas é elevada, não tendo sido possível, a nível internacional, atingir um entendimento consensual sobre investigação de qualidade, ou sobre os atributos que possibilitam a aferição dessa qualidade. Assim, embora os estudos em Ciências Sociais e Humanas tendam a perfilhar um enfoque contextualista, na qual os dados são interpretados tendo como propósito a compreensão profunda de problemas e situações 
concretas, na contemporaneidade, a investigação assume uma elevada multiplicidade de orientações epistemológicas, ontológicas e metodológicas. Desta breve sinopse seguiu-se a revisão de três linhas distintas de sistematização critérios de apreciação da qualidade da investigação científica, quando esta se consubstancia em tradições não positivistas.

Caberá ao/à leitor/a a seleção daquela(s) que lhe pareça(m) mais ajustada(s) para o seu esforço de avaliação da qualidade dos estudos científicos, responsabilizando-se, nesta escolha, pela valorização de determinados elementos em detrimento de outros, que poderão ser descurados ou mesmo invisibilizados se a opção for demasiado restritiva para fazer face à complexidade da pesquisa alvo de apreciação.

\section{Referências}

AMADO, J. Manual de investigação qualitativa em educação. 1. ed. Coimbra: Imprensa da Universidade de Coimbra, 2013.

BOGDAN, R.; BIKLEN, S. Investigação qualitativa em educação: Uma introdução à teoria e aos métodos. 2. ed. Porto: Porto Editora, 2013.

CORBIN, J.; STRAUSS, A. Grounded Theory research: Procedures, cannons, and evaluative criteria. Qualitative Sociology, New York, v. 13, n. 1, p. 3-21, 1990.

DAVIES, D.; DODD, J. Qualitative research and the question of rigor. Qualitative Health Research, London, v. 12, n. 2, p. 279-289, fev. 2002.

DENZIN, N.; LINCOLN, Y. (Eds.). Handbook of qualitative research. 2. ed. Thousand Oaks, CA: Sage, 2000.

FERREIRA, S. À luz da lua, na face negra da terra. 2015. 853 p. Tese (Doutorado em Ciências da Educação) - Faculdade de Psicologia e de Ciências da Educação, Universidade de Coimbra, Coimbra, 2015.

FEYERABEND, P. Contra o método. 1. ed. Lisboa: Relógio d'Água, 1993.

FEYNMAN, R. What is science. The Physics Teacher. Maryland, v. 7, n. 6, p. 313-320, Sept. 1969.

HESSE-BIBER, S.; LEAVY, P. (Eds.). Approaches to qualitative research. 1. ed. New York: Oxford University Press, 2004.

GLASER, B.; STRAUSS, A. Awareness of dying. 1. st. ed. Chicago, IL: Aldine Publishing Company, 1965.

GLASER, B.; STRAUSS, A. The discovery of grounded theory: Strategies for qualitative research. 1. st. ed. Chicago, IL: Aldine Publishing Company, 1967.

GLAZIER, J.; POWELL, R. (Eds.). Qualitative research in information management. 1. st. ed. Englewood, CO: Libraries Unlimited, 1992.

GOLAFSHANI, N. Understanding reliability and validity in qualitative research. The

Qualitative Report, Fort Lauderdale, v. 8, n. 4, p. 597-607, Dec. 2003. 
GUBA, E. G.; LINCOLN, Y. S. Competing paradigms in qualitative research. In N. K. Denzin; Y. S. Lincoln (Eds.). The landscape of qualitative research: Theories and issues. 1. st. ed. p.195-220. Thousand Oaks, CA: Sage, 1998.

HOPE, K.; WATERMAN, H. Praiseworthy pragmatism? Validity and action research. Journal of Advanced Nursing, Malden, v. 44, n. 2, p. 120-127, Oct. 2003.

LAW, J. After method: Mess in social science research. 1. ed. New York: Routledge, 2006.

LINCOLN, Y.; GUBA, E. Naturalistic inquiry. 1. st. Newbury Park, CA: Sage, 1985.

MERTENS, D. Research methods in education and psychology: Integrating diversity with quantitative and qualitative approaches. 1. ed. Thousand Oaks, CA: Sage, 1998.

PARTINGTON, G. Choosing schools. Quadrant, Melbourne, v. 48, n. 11, p. 56-60, Nov. 2004.

PATTON, M. Qualitative evaluation and research methods. 3. ed. Thousand Oaks, CA: Sage, 2001.

RAGIN, C. Redesigning social inquiry: Fuzzy sets and beyond. 1. ed. Chicago, IL: University of Chicago Press, 2008.

ROLFE, G. Validity, trustworthiness and rigour: Quality and the idea of qualitative research. Journal of Advanced Nursing, Malden, v. 53, n. 3, p. 304-310, Jan. 2006.

SANDELOWSKI, M. Rigor or rigor mortis: The problem of rigor in qualitative research revisited. Advanced Nursing Science, Philadelphia, v. 16, n. 2, p. 1-8, May. 1993.

SANDELOWSKI, M.; BARROSO, J. Finding the findings in qualitative studies. Journal of Nursing Scholarship, Malden, v. 34, n. 3, p. 213-219, Sept. 2002.

SILVERMAN, D. A very short, fairly interesting and reasonably cheap book about qualitative research. 2nd ed. Thousand Oaks, CA: SAGE, 2013.

STENBACKA, C. Qualitative research requires quality concepts of its own. Management Decision, Bingley, v. 39, n. 7, p. 551-555, 2001.

STRAUSS, A.; CORBIN, J. Basics of qualitative research: Techniques and procedures for developing grounded theory. 2. ed. Thousand Oaks, CA: Sage, 1998.

WAGNER, S.; LUKASSEN, P.; MAHLENDORF, M. Misused and missed use: Grounded theory and objective hermeneutics as methods for research in industrial marketing. Industrial Marketing Management, Radarweg, v. 39, n.1, p. 5-15, Sept. 2010.

WINTER, G. A comparative discussion of the notion of validity in qualitative and quantitative research. The Qualitative Report, Fort Lauderdale, v. 4, n. 3/4, p. 1-14, jan. 2000. Disponível em: < http://www.nova.edu/ssss/QR/QR4-3/winter.htm.l>. Acesso em: 25 maio 2016.

Recebido em: 14 de dezembro de 2017.

Aceito em: 24 de outubro de 2018. 